\title{
Evolution of Epidural Lysis of Adhesions
}

\author{
Susan R. Anderson, MD*, Gabor B. Racz, MD², and James Heavner, DVM, PhD**
}

Epidural neuroplasty (lysis of epidural adhesions) is an interventional technique that has emerged over the last 10 years as part of a multidisciplinary approach to treating radiculopathy with low back pain when conservative management has failed. Neuroplasty was at one time performed as a single-catheter technique using the caudal approach. It now has many variations, including placement of the catheter tip in the anterior epidural space. This article will discuss the evolution and refinement of epidural neuroplasty at our institution.

Keywords: Low back pain, radiculopathy, epidural, local anesthetic, corticosteroid, hyaluronidase, hypertonic saline, neuroplasty
Epidural neuroplasty (lysis of epidural adhesions) is an interventional technique that was developed at Texas Tech Health Sciences Pain Center in 1989. It is indicated when conservative management for spinal or radicular pain has failed. Indications include failed neck/back surgery syndrome, disc disruption, vertebral compression fractures, multilevel degenerative arthritis, facet pain, epidural scarring, and pain not entirely responsive to spinal cord stimulation or intrathecal opioids. This article discusses the evolution and refinement of epidural neuroplasty at our institution over the last 10 years.

\section{ANATOMY AND PHYSIOLOGY OF LOW BACK PAIN WITH AND WITHOUT RADICULOPATHY}

The determination of which tissues contribute to the origin of back pain was confirmed when Kuslich and colleagues (1) performed 193 operations on the lumbar spines of patients given local anesthesia for surgery. They demonstrated that sciatica could only be produced by stimulation of a swollen, stretched, restricted, ie, scarred, or compressed nerve root. Back pain could be produced by stimulation of several lumbar tissues, but the most common

From Department of Anaesthesiology Texas Tech University Health Sciences Center, Lubbock, Texas. * Dr. Susan R. Anderson is assistant professor, ${ }^{\boldsymbol{\Omega}}$ Dr. Gabor B. Racz is professor and chairman emeritus, and ** Dr. James E. Heavner is professor. Address Correspondence: Susan R. Anderson, MD, Department of Anesthesiology, Texas Tech University Health Sciences Center, 3601 4th Street, Room 1C-275, Lubbock, TX 79430 tissue of origin was the outer layer of the annulus fibrosus and posterior longitudinal ligament. Stimulation for pain generation of the facet-joint capsule rarely generated low back pain, and facet synovium and cartilage surfaces of the facet or muscles were never tender (2).

The contribution of fibrosis as the origin of low back pain has been debated. In patients who had undergone prior laminectomies, there was always some degree of perineural fibrosis. Although scar tissue itself was never tender, the nerve root was frequently very sensitive. Kuslich (1) concluded that the presence of scar tissue compounded pain associated with the nerve root by fixing it in one position and thus increasing the susceptibility of the nerve root to tension or compression. Moreover, these researchers concluded that, "Sciatica can only be reproduced by direct pressure or stretch on the inflamed, stretched, or compressed nerve root. No other tissues in the spine are capable of producing leg pain.” Stolker and coworkers (3) contended that innervated structures are the only originators of pain.

Mechanical factors are not the only causative factors of radicular pain. Histological injury may occur without compression, resulting in persistence of radicular symptoms. Nerve roots may be exposed to chemical irritant substances from degenerated intervertebral discs or facet joints, which can generate pain $(2,4)$. Structures in the ventral epidural space may become highly sensitized by chemical irritation, resulting in axial pain. These structures include (5):
1. Ventral dura,
2. Posterior longitudinal ligament,
3. Vertebral periosteum, 
4. Dural attachments, and

5. Epiradicular components.

Innervation to the ventral epidural space is extensive and, thus, may become highly sensitized, resulting in chronic low back pain. Histopathological studies have demonstrated sinuvertebral nerve and sympathetic innervation (6-8).

Pressure-induced changes in the nerves depend upon whether the compression pressures are high or low, e.g. < $200 \mathrm{~mm}$ Hg. High pressures exert a direct mechanical effect that can result in deformation of nerve fibers, displacement of the nodes of Ranvier, or invaginations of the paranodal myelin sheaths (2). Low pressures may lead to impairment of blood supply to tissue, decreased nutrient transport, and intraneural edema. Olmarker and colleagues (4) demonstrated that blood flow in the cauda equina ceases when applied pressure (in this case an inflated balloon fixed to the spine in animals) equals mean arterial pressure. A pressure of 5 to $10 \mathrm{~mm} \mathrm{Hg}$ stopped blood flow in some venules $(2,4)$. An applied pressure of $10 \mathrm{~mm} \mathrm{Hg}$ decreased the nutrient transport to nerve roots by $20 \%$ to $30 \%$ (4). Compression may also lead to changes in permeability or transmural pressure of endoneural capillaries. This can produce edema. It has been demonstrated that intraneural edema in chronic nerve injury is related to the formation of intraneural fibrosis (2).

Chemical irritation is another factor in the origin of back pain with or without radiculopathy. The nucleus pulposus has been identified as a source of chemicals which, when leaked into the spinal canal via a rent in the annulus fibrosus, produce irritation. Nerve roots compressed by disc material often show signs of inflammation. Substances that might leak from the disc and produce inflammation of nerve roots or meninges include lactic acid, glycoprotein, cytokines, and histamine. In addition, it has been theorized that material from the nucleus pulposa might act as a foreign protein and trigger an autoimmune reaction (4). Obviously, chemically induced irritation can occur in the absence of compression by the disc (2).

Structural changes in the vertebral column provide other contributing factors. In a review of the literature, Benzon (9) pointed out that abnormalities of the intervertebral disc include degeneration, bulging, and herniation. Narrowing of the disc space as a consequence of disc pathology is frequently associated with osteophyte formation, and osteoarthritis of the facet joints, which can put pressure on spinal nerves. Bulging of the intervertebral disc distends the posterior longitudinal ligament, causing lo-

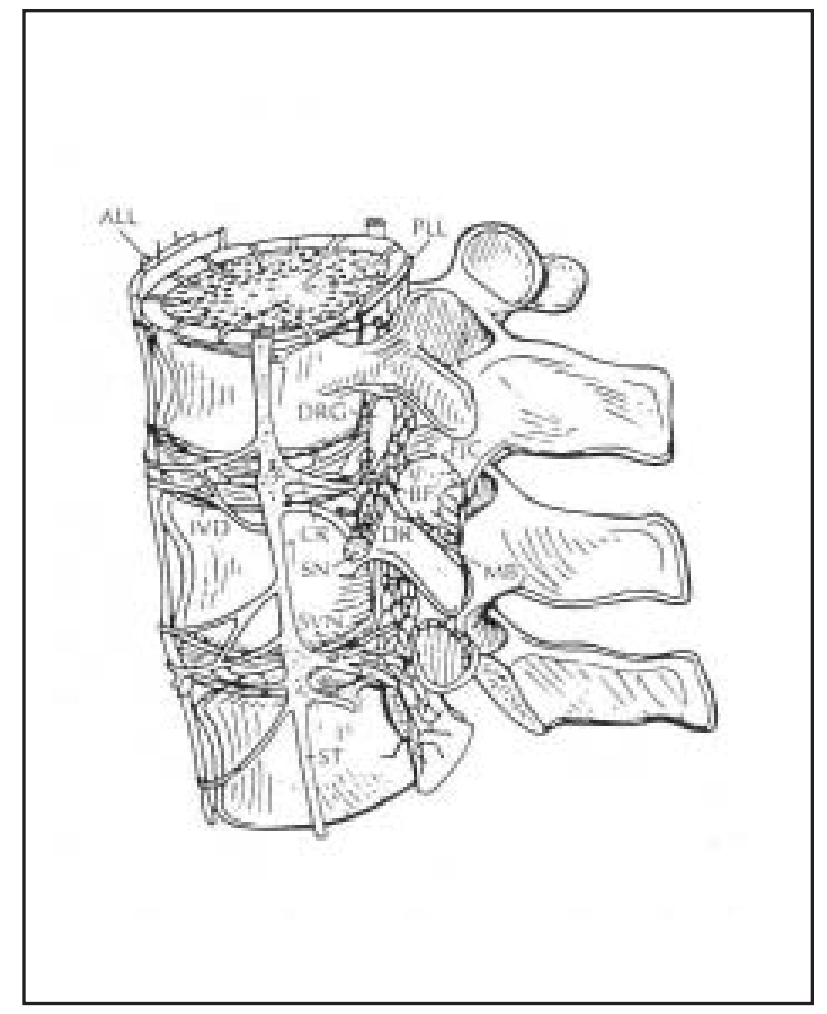

Fig. 1. Schematic drawing of the nerve supply of the spine. The ventral compartment of the spine is supplied by interconnected nerve plexuses derived from branches from the sympathetic trunk and from meningeal rami of the spinal nerves, ie, the sinuvertebral nerves. The sinuvertebral nerves have their origin in the rami communicans. The dorsal compartment is supplied by the dorsal rami of the spinal nerves and contains brances from the rami communicans as well. All - anterior longitudinal ligament; BF - fibers from the communicating ramus bypassing the spinal nerve and joining the dorsal ramus; CR - communicating ramus; DR - dorsal ramus of the spinal nerve; DRG - dorsal root ganglion; FJC - facet-joint capsule; IVD - intervertebral disc; MB - medial branch of the dorsal ramus; PLL - posterior longitudinal ligament; SN - sinuvertebral nerve. (From Stolker and colleagues) (3).

calized back pain. If bulging of the disc increases, pressure may be exerted on the adjacent nerve roots, producing radicular pain (2).

Innervation of the structures of the spine was reviewed by Stolker and colleagues (Fig. 1) (3). Relevant to epidural neurolysis is the fact that free nerve endings have been demonstrated in structures identified by Kuslich and coworkers (1) to be involved in low back pain, ie, the posterior longitudinal ligament and the annulus fibrosus. Interconnected neural networks supply the anterior and posterior longitudinal ligaments, and ventral dura. Perivas- 
cular nerves innervating the annulus fibrosus bilaterally and multisegmentally (three to five levels) contribute to formation of the neural plexus of the anterior longitudinal ligament (10). The posterior longitudinal ligament plexus consists of branches of the communicating rami, which re-enter the vertebral canal as sinuvertebral nerves. This plexus supplies, bilaterally and multisegmentally (two to four levels), the posterior part of the annulus fibrosus and the vertebral body. The anterior and posterior longitudinal ligament plexus is interconnected by branches of the communicating rami (11).

Although considerable debate exists as to whether epidural fibrosis causes pain $(12,13)$, it is widely accepted that postoperative scar tissue renders the nerve susceptible to injury via compression phenomena, ie, stenosis, disc, etc. (14). Scar tissue is generally found in three components of the epidural space. Dorsal epidural scar tissue is formed by resorption of surgical hematoma and may be involved in pain generation (15). In the ventral epidural space, dense scar tissue is formed by ventral defects in the disc, which may persist despite surgical treatment and continue to produce chronic low back pain/radiculopathy past the surgical healing phase (16). The lateral epidural space includes epiradicular structures out of the root canals known as "sleeves" containing the exiting nerve root and dorsal root ganglia, susceptible to lateral disc defects, facet overgrowth, neuroforaminal stenosis, etc. (4).

Based on the above discussion of possible pain generators, one or all of the following pathological changes may be present in patients with chronic low back pain with or without radiculopathy. These changes include: inflammation, edema, fibrosis, venous congestion, mechanical pressure on the posterior longitudinal ligament, reduced or absent nutrient delivery to the spinal nerve or nerve root, and central sensitization. Inflamed tissue may generate activity in nociceptors or axons that convey nociceptive information to the CNS. Inflammation may render nociceptors or nociceptor axons more sensitive to mechanical stimuli (2).

\section{EVOLUTION OF THE TECHNIQUE}

When the technique of epidural neuroplasty (lysis of epidural adhesions) was first developed, the catheter was inserted into the posterior epidural space. As more and more procedures were performed, it was noted that the posterior epidural space was difficult to access in some patients who had undergone surgical procedures for discogenic or radicular pain. This was most likely due to scar tissue from the previous surgery. In 1996, the technique for catheter insertion was changed to directing the catheter into the anterior epidural space. The catheter needed to be directed towards the anterior epidural space at the level of the S3 nerve root on the affected side for caudal neuroplasty. It also required the open end of the epidural needle to be directed anterolateral after insertion through the sacral hiatus.

\section{PROPOSED ALGORITHM}

Based on anatomy and pathological changes, an algorithm for the treatment of low back pain with and without radiculopathy may be proposed, with the rationale of local application of medications at the site of the pathology (Fig. 2). Workup of a patient begins with a thorough history, clinical exam, and diagnostic workup. The effect of failed conservative therapies should be noted. These treatment modalities include physical therapy, minor nerve blocks, transcutaneous electrical nerve stimulation, behavioral medicine, nonsteroidal anti-inflammatory medications, membrane stabilizers, and antidepressants. Also, prior to neuroplasty, all attempts should be made to eliminate nociceptive and sympathetically maintained pain. To treat the patient with back pain with and without radiculopathy, the pain-generating components must be addressed separately. The algorithmic approach can be used after all conservative therapies have failed. It is imperative that the patient has ongoing concurrent and physical therapy, functional restoration, psychotherapy, and pharmacological management. An algorithm for the treatment of back pain includes recognition and treatment of myofascial pain, facet arthropathy, and internal disc

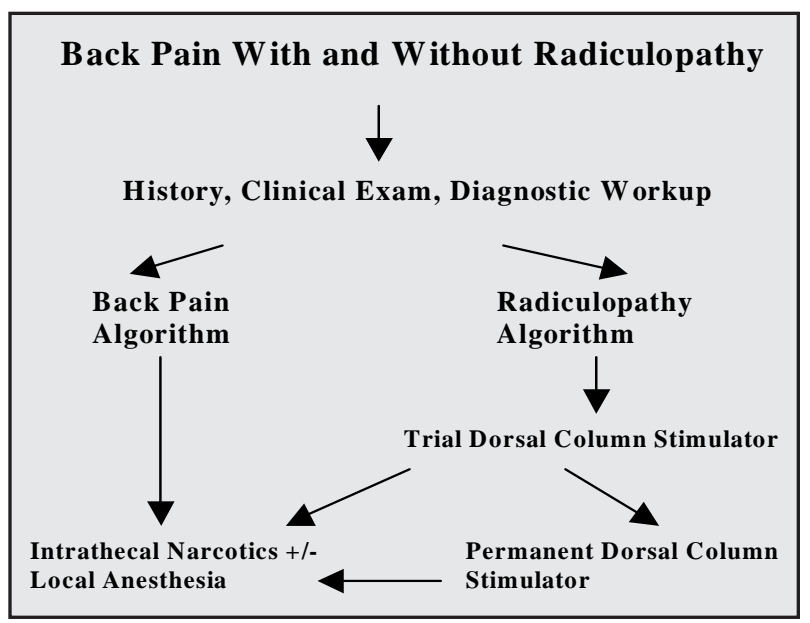

Fig. 2. Overall view of the algorithm for treatment of back pain with and without radiculopathy. 


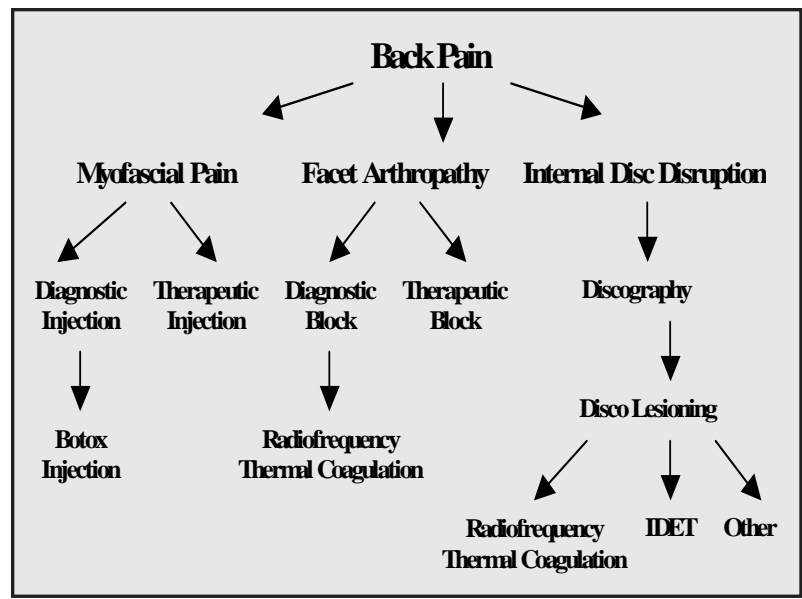

Fig. 3. Algorithm for treatment of back pain.

disruption (Fig. 3). The approach for radiculopathy includes treatment for epidural fibrosis, spinal fibrosis, and primary or secondary discogenic pain with epidural steroid injections or selective nerve-root-sleeve injections (Figs. 4 and 5). It should be noted that neuroplasty is appropriate after a failed single-shot epidural steroid injection trial. Often this is due to accumulation of fibrotic tissue in the epidural space that prevents spread of the medication to the site of pathology (Fig. 4). Further, if a significant filling defect is evident with placement of the caudal catheter for neuroplasty, a second catheter should be placed transforaminally. Finally, a single-catheter technique with epiduroscopy is being developed that will include neurophysiologic mapping. It should be noted that implantable techniques such as intrathecal narcotic pumps and dorsal column stimulators are considered after all

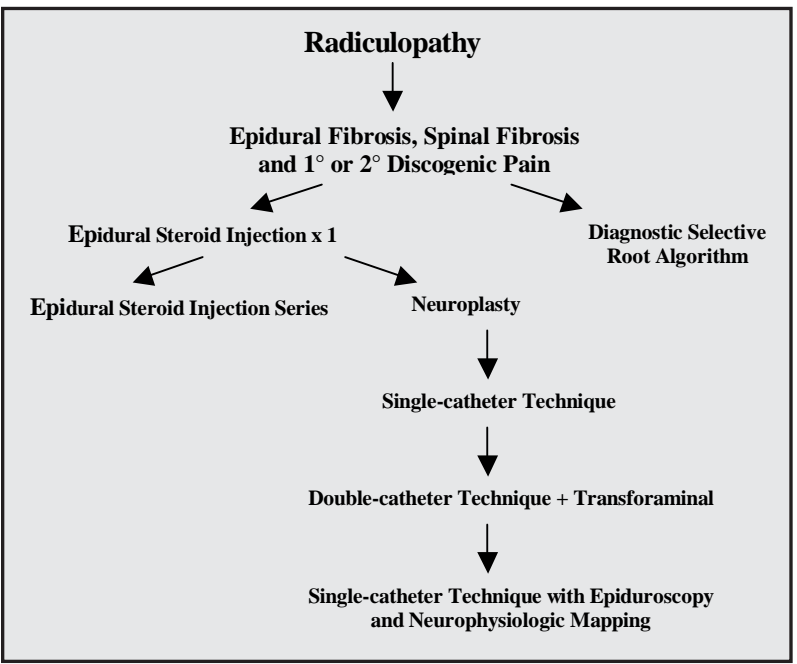

Fig. 4. Algorithm for treatment of radiculopathy with epidural steroids.

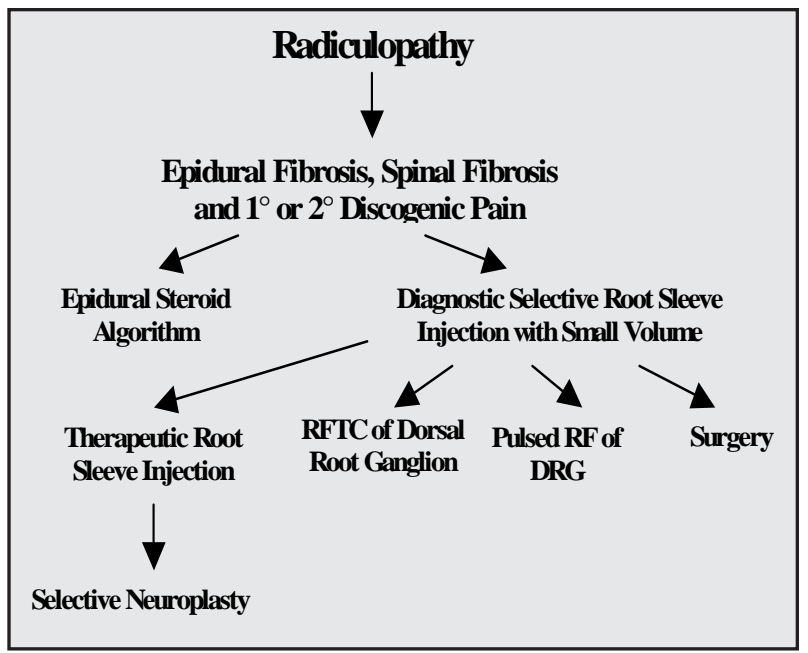

Fig. 5. Algorithm for treatment of radiculopathy with selective root-sleeve injections.

other therapies have failed.

\section{PREPARATION OF THE PATIENT}

The history and physical exam should be thorough and note failed conservative therapies. It is important to delineate chronic low back pain with or without radiculopathy as seen secondary to failed spinal surgery or chronic low back pain secondary to internal disc disruption. The physical exam should include neural tension tests, neurological changes, and maneuvers that provoke axial pain. Specific neural tension tests are the straight-leg raise from 30 to 60 degrees and the slump test. The slump should note provocation with proximal and/or distal initiation. The neurological testing should reveal dermatomal or myotomal loss, reflex changes, and motion changes. The diagnostic workup includes radiographic and physiologic exams. Xrays of the lumbar spine in flexion and extension may reveal a hypermobility problem. A magnetic resonance imaging scan with gadolinium is necessary to demonstrate epidural fibrosis. Discography may reveal an internal disc disruption. Physiologic studies might include electromyograph/nerve conduction studies or somatosensory-evoked potentials.

Prior to an invasive procedure such as neuroplasty, other issues must be addressed. The patient must be willing to participate in a multidisciplinary rehabilitation program. Significant psychopathology must be ruled out with psychological testing and evaluation. Secondary gain issues, be they monetary, related to medication, or psychological, must be addressed. Finally, detoxification from narcotics, if an issue, must be accomplished. 


\section{COMPLICATIONS}

A number of complications may be encountered when performing neuroplasty, whether it be by the caudal, thoracic, cervical, or transforaminal route. These include subarachnoid or subdural injection of local anesthetic or hypertonic saline, paralysis, bowel and/or bladder dysfunction, spinal cord compression from loculation of the injected fluids or hematoma, infection, sensitivity to hyaluronidase, and catheter shearing.

\section{DRUGS USED FOR NEUROPLASTY}

The medications used in neuroplasty are directed at the origination of pain generators (7). Iohexol, the nonionic, water-soluble contrast dye, is used to identify filling defects for placement of the catheter. It is low in osmolarity for decreased toxicity. Iohexol is renally excreted in unmetabolized form (88\% intrathecal dose detectable in urine within 24 hours) (17). Chemotoxic reactions are 1/ 100,000 in occurrence. The dose-dependent reaction includes sudden hypotension, organ failure, cardiac arrest, and loss of consciousness. Idiosyncratic reactions include headache (18\%) (17), myalgias, nausea, vomiting, dizziness, aseptic meningitis, and allergic, or anaphylactoid reactions (18). The risk for injection into the epidural space is considered no more significant than with diagnostic lumbar puncture (19). Ropivacaine is the local anesthetic used to block nerve axons. It is an amide similar to lidocaine, mepivacaine, or bupivacaine. Advantages of ropivacaine as compared to bupivacaine are: shorter time to onset, shorter duration of motor block, a less cardiotoxic profile, and more selective action on Adelta and C fibers (20). Corticosteroids are injected as an anti-inflammatory agent and for reduction of edema. Triamcinolone diacetate has high glucocorticoid activity (21) with related side effects, e.g., muscle wasting, peptic ulcer, impaired wound healing, and impaired immunologic function (22). It is preservative free and, more importantly, does not flocculate with lidocaine as methylprednisolone does (Antal EG. Pharmacia, and Upjohn, Inc., Kalamazoo, MI, written communication, July 3, 1997). It does suppress the hypothalamic-pituitary-adrenal axis for 21 days (23). Triamcinolone also has nonglucocorticoid-related side effects such as allergic reactions, arachnoiditis from intrathecal injections (24), meningitis (aseptic and bacterial), and epidural abscess $(25,26)$. Hyaluronidase is injected to disrupt the ground substance in epidural adhesions. This leads to accelerated diffusion (27) and increased absorption by increasing capillary permeability $(28,29)$. It is noted to pre- serve the dura and provides improved efficacy of decompressive neuroplasty (3). Hypertonic saline decreases tissue edema and increases the fluid volume within the epidural space, causing microdissection. Hypertonic saline is also noted to have some local anesthetic action $(30,31)$ and C-fiber selectivity (32). A possible complication from injection of hypertonic saline is arachnoiditis (33).

\section{TECHNIQUE}

Epidural neuroplasty is performed in the caudal region for low back pain. It can also be performed in the cervical and thoracic areas with modifications, ie, the technique for catheter insertion and the different dosages of medications used. When the procedure is chosen, it should be thoroughly explained to the patient, including all benefits and risks. Informed consent should be obtained. A preoperative complete blood cell count, prothrombin time, partial thromboplastin time, bleeding time, and urinalysis are required. Laboratory values should be optimal.

\section{PROTOCOL}

\section{Caudal Approach}

In the operating room:

1. Routine intraoperative monitors are placed, an intravenous drip is started, and appropriate sedation is given. Place the patient in the prone position. Place sterile drapes in position.

2. Place a 15- or 16-gauge $\mathrm{RK}^{\mathrm{TM}}$ epidural needle via the sacral hiatus on the side opposite the suspected pathology after local anesthetic infiltration of the skin on the top of the buttock.

3. With confirmation of the correct placement of the needle in the epidural space, inject $10 \mathrm{~mL}$ iohexol (Omnipaque $240^{\mathrm{TM}}$ ) after negative aspiration and visualize spread of the contrast medium (epidurogram).

4. If a filling defect corresponding to the area of pain is present, thread a Racz Tun-L-Kath ${ }^{\mathrm{TM}}$ or Racz Tun-L-XL ${ }^{\mathrm{TM}}$ (stiffer) catheter towards the filling defect (scarring or fibrosis). Prior to insertion, place a 30-degree bend approximately $2.5 \mathrm{~cm}$ from the catheter tip to aid in placement of the catheter towards the side and anteriorly. Confirm ven- 
tral placement of the catheter with a lateral fluoroscopic view.

5. After correct placement, inject $10 \mathrm{~mL}$ of preservative-free normal saline with $1500 \mathrm{U}$ of hyaluronidase into the filling defect.

6. Inject an additional 2 to $3 \mathrm{~mL}$ of iohexol to visualize opening of the scarred area and spread of the injectate within the epidural space.

7. Inject $9 \mathrm{~mL}$ of $0.2 \%$ ropivacaine with 1 $\mathrm{mL}$ of $40 \mathrm{mg} / \mathrm{mL}$ triamcinolone diacetate. First inject a 3-mL test dose, followed 5 minutes later by the remaining $7 \mathrm{~mL}$ if there is no evidence of intravascular or intrathecal injection.

8. Remove the needle and secure the catheter to the skin with 3.0 nylon suture. Apply triple-antibiotic ointment to the skin entry site and place a sterile dressing.

9. Attach a bacteriostatic filter to the catheter.

In the postanesthesia care unit (PACU) 30 minutes later:

1. If there is no evidence of a subarachnoid block, infuse $10 \mathrm{~mL}$ of $10 \%$ saline via the catheter over 30 minutes. If the patient complains of pain, stop the infusion and inject an additional 2 to 3 $\mathrm{mL}$ of $0.2 \%$ ropivacaine via the catheter, wait 5 minutes and restart the infusion.

2. At the end of the infusion, flush the catheter with $2 \mathrm{~mL}$ of preservative-free normal saline.

In the PACU (or designated procedure area) after 18 to 24 hours:

1. On the following 2 days, inject $10 \mathrm{~mL}$ of $0.2 \%$ ropivacaine; wait 25 minutes, infuse $10 \mathrm{~mL}$ of $10 \%$ saline over 30 minutes.

2. Flush catheter with $2 \mathrm{~mL}$ of preservative-free normal saline.

3. On day 3 , repeat the above process, remove the catheter, and place sterile dressing.

\section{Three D Technique}

For epidural neuroplasty in the cervical and thoracic region, the approach to accessing the epidural space uti- lizes the "Three $D$ ” technique. This involves using fluoroscopic views to ascertain Direction, Depth, and Direction of the epidural needle as one approaches the epidural space. The synopsis of the technique for cervical and thoracic neuroplasty follows.

In the operating room:

1. Position the patient in the left lateral decubitus position.

2. Use the paramedian approach $1 \mathrm{~cm}$ from midline, one interspace below the intended epidural space entry.

3. Obtain epidural access with a 16-gauge RK epidural needle.

4. Use anteroposterior fluoroscopy to assess direction.

5. Use lateral fluoroscopy to assess depth.

$6 . \quad$ Use anteroposterior fluoroscopy to reassess direction and correct if necessary.

7. Advance the needle to the base of the spinous process that corresponds to the level of the lamina near the posterior aspect of the epidural space. The tip of the needle should be midline. Remove the stylet.

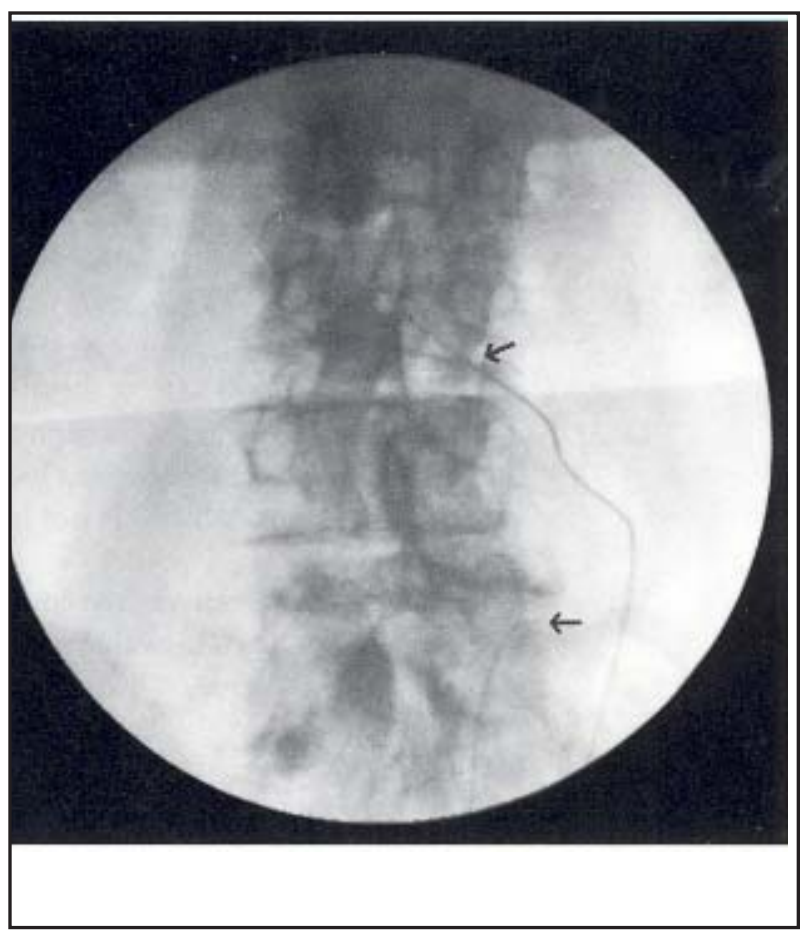

Fig. 6. Anteroposterior view of placement of the transforaminal catheter (top arrow) and caudal catheter (bottom arrow) for the double-catheter technique. 


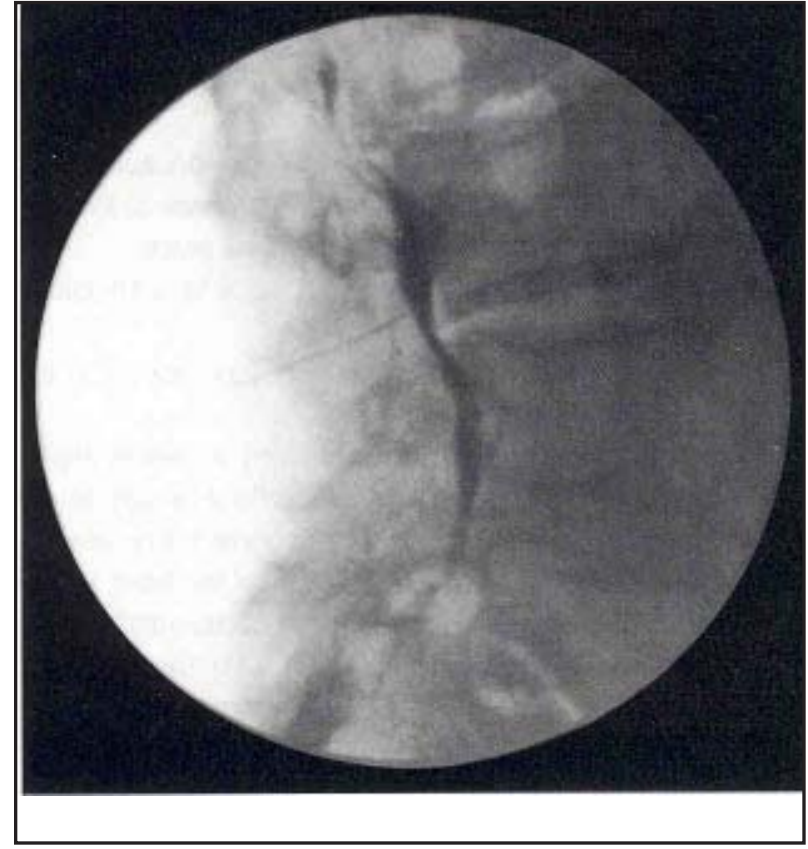

Fig. 7. Lateral view of correct placement of the transforaminal catheter with confirmation by dye spread.

8. Using loss-of-bounce technique with a pulsator syringe containing $4 \mathrm{~mL}$ of normal saline and $2 \mathrm{~mL}$ of air, enter the epidural space.

9. $\quad$ Perform an epidurogram by injecting 2 to $5 \mathrm{~mL}$ of iohexol through the needle.

10. Thread the Racz Tun-L-Kath ${ }^{\mathrm{TM}}$ through the needle toward the targeted nerve root in the lateral epidural space; lateral placement is optimal.

11. Inject 1 to $2 \mathrm{~mL}$ of iohexol through the catheter to verify that there are no loculations and that the catheter is not in a blood vessel.

12. Inject $6 \mathrm{~mL}$ of preservative-free normal saline containing 1500 U hyaluronidase if in the cervical region, $8 \mathrm{~mL}$ if in the thoracic region.

13. If in the cervical region, inject $5 \mathrm{~mL}$ of $0.2 \%$ ropivacaine with $1 \mathrm{~mL}$ of $40 \mathrm{mg} /$ $\mathrm{mL}$ triamcinolone diacetate $(6 \mathrm{~mL}$ total volume) in 2 to $3 \mathrm{~mL}$ incremental doses to verify that the catheter is not subdural or subarachnoid. If in the tho- racic region, the total volume is $8 \mathrm{~mL}$, including the steroid.

14. Remove the needle under fluoroscopic guidance and suture the catheter in place with 3.0 nylon suture.

15. Attach the connector to the catheter with a bacteriostatic filter.

16. Apply triple-antibiotic ointment and cover catheter with split $2 \times 2 s$ and a transparent dressing. Place a half loop in the catheter prior to applying the transparent dressing. Tape the catheter to the patient's skin.

In the PACU:

1. If there is no evidence of subdural or subarachnoid spread, infuse the catheter with 4 to $5 \mathrm{~mL}$ of $10 \%$ saline for the cervical region and $8 \mathrm{~mL}$ if in the thoracic region. The infusion should run in over 30 minutes.

2. Flush the catheter with $2 \mathrm{~mL}$ of preservative-free normal saline.

In the PACU or designated procedure area:

1. On days two and three, aspirate the catheter and inject $6 \mathrm{~mL}$ (cervical) or 8 $\mathrm{mL}$ (thoracic) of $0.2 \%$ ropivacaine over 25 minutes. First give a $2-m L$ test bolus, wait 5 minutes as a precaution against subarachnoid or subdural spread, and then give the remaining amount over 20 minutes.

2. Infuse 4 to 5 or $8 \mathrm{~mL}$ (cervical or thoracic, respectively) of $10 \%$ saline over 30 minutes.

3. Flush with $2 \mathrm{~mL}$ of preservative-free normal saline.

4. On day three, remove the catheter at the end of the infusion and apply a sterile dressing.

\section{Transforaminal Approach}

Recently a lumbar transforaminal technique has been advocated for patients for whom the area of scarring is above the L5/S1 nerve root, making it difficult to access via the caudal approach. Using a 15-degree oblique and a 15degree caudocephalad fluoroscopic view, a curved $\mathrm{RK}^{\mathrm{TM}}$ epidural needle is directed using gun-barrel technique towards a point just lateral to the superior pars of the vertebrae that comprises the caudad portion of the neural foramen. The tip of the needle should be 2 to $3 \mathrm{~mm}$ within 
the neural foramen. Two to three milliliters of contrast is injected to confirm epidural spread. The Tun-L-Kath or Tun-L-XL ${ }^{\mathrm{TM}}$ is inserted through the needle into the epidural space. If resistance is met, advance the needle 1 to $2 \mathrm{~mm}$ more and reinsert the catheter. (A guidewire or a stiff probe with a flexible, blunt or atraumatic tip may be inserted through the needle to facilitate opening of the anterior epidural space.) Once inside the epidural space, the catheter is advanced to the midline and the epidural needle is removed (Figs. 6 and 7). The injection of medications follows the same guidelines as those for caudal neuroplasty. This approach may be used in conjunction with a caudal catheter for a double-catheter technique, when deemed necessary.

\section{OUTCOMES}

The first report of this procedure was by Racz and Holubec in 1989 (34). The results were based on a survey of 72 patients who were randomly selected from a pool of approximately 200 patients who underwent caudal neuroplasty. There were slight variations in the protocol, namely the dose of bupivacaine used $(25 \mathrm{~mL}$ instead of $10 \mathrm{~mL}$ ) and the omission of hyaluronidase. Approximately $72.2 \%$ of the patients reported pain relief on discharge, while $37.5 \%$ had pain relief of $<1$ month, $30.0 \%$ reported pain relief of 1 to 3 months' duration, and $12.5 \%$ had relief for 3 to 6 months. Arthur, Racz, Heinrich and colleagues (35) presented another retrospective study that used the same technique, except only 50 of the 100 patients received hyaluronidase. In the hyaluronidase group, $81.6 \%$ of the patients had persistent pain relief at the time of interview (in some up to 3 years postprocedure). In the no-hyaluronidase group, $68 \%$ of the patients had varying degrees and durations of pain relief, and 15\% had persistent pain relief. Most recently, Racz, Raj, and Heavner (36) performed a prospective evaluation of $0.9 \%$ sodium chloride versus $10 \%$ sodium chloride with or without hyaluronidase. Results from this study show that $49 \%$ of the patients had pain relief in the body area targeted for lesion-specific therapy at 1-year follow-up. Hammer and coworkers performed a study on the double catheter (caudal and transforaminal) technique with findings in agreement with Texas Tech University (Hammer. M, Transforaminal Lumbar Epidural Neuroplasty, unpublished data, 2000). After 2 weeks, patients reported a $50 \%$ or greater relief of pain. At 6 months' follow-up, $86 \%$ of patients would repeat the procedure, $60 \%$ attained more than $50 \%$ pain relief, $10 \%$ achieved $100 \%$ pain relief, $20 \%$ achieved less than $50 \%$ pain relief, and $10 \%$ of patients had no change in their pain severity (12).
Manchikanti and colleagues, in a retrospective study, (37) report that a 1-day injection series is as effective as the 3day injection series per Racz and coworkers. Hence, a prospective study for the 1-day injection series should be done.

\section{SUMMARY}

Percutaneous epidural neuroplasty has been proven to be safe and effective in selected patients with epidural adhesions. It must be performed by a technically competent, trained pain physician. Recent information on the innervation of the ventral epidural space emphasizes the need for ventral, site-specific catheter placement for delivery of medications (2). It is imperative to adhere to the technique (including the placement of the catheter tip into the scar) without modification for maximizing benefits. The prospective studies indicate improved and cost-effective results $(2,37,38)$.

\section{REFERENCES}

1. Kuslich SD, Ulstrom CL, Michael CJ. The tissue origin of low back pain and sciatica. Orthopaedic Clin NA 1991; 22:181-187.

2. Racz GB, Noe C, Heavner JE. Selective spinal injections for lower back pain. Current Review of Pain 1999; 3:333-341.

3. Stolker RJ, Vervest ACM, Groen GJ. The management of chronic spinal pain by blockades: A review. Pain 1994; 58:1-20.

4. Olmarker K, Rydevik B. Pathophysiology of sciatica. Orthopedic Clin NA 1991; 22:223-233.

5. Cautico W, Parker JC, Pappert E et al. An anatomical and clinical investigation of spinal meningeal nerves. Aeta Neurochir (Wien) 1988; 90:139-143.

6. Imai S, Hukuda S, Maeda T. Dually nnervating nociceptive networks in the rat lumbar posterior longitudinal ligaments. Spine 1995; 19:2086-2092.

7. Lewandowski EM. The efficacy of solutions used in caudal neuroplasty. Pain Digest 1997; 7:323-330.

8. Olmarker K, Rydevik B, Nordburg C. Autologous nucleus pulposus induces neurophysiologic and histologic changes in porcine cauda equina nerve roots. Spine 1993; 11:1425-1432.

9. Benzon HT. Epidural steroid injection for low back pain and lumbosacral radiculopathy. Pain 1986; 24:277-295.

10. Groen GJ. De innervatie van de wervelkolom bij de mens. Ned Tijdschr Man Ther 1991; 10:48-60.

11. Groen GJ, Baljet B, Rdukker J. Nerves and nerve plexus of the human vertebral column. Am J Anat 1990; 188:282-296.

12. Gaughan E, Nikon A, Krooh L et al. Effect of sodium 
hyaluronate on tendon healing and adhesion formation in the horse. Am J Vet Res 1991; 52:764-773.

13. Annertz M, Jonsson B, Stromqvist B et al. No relationship between epidural fibrosis and sciatica in the lumbar postdiscectomy syndrome. A study with contrast-enhanced magnetic resonance imaging in symptomatic and asymptomatic patients. Spine 1995; 4:449-453.

14. Cervellini P, Curri D, Volpin L et al. Computed tomography of epidural fibrosis after discectomy. A comparison between symptomatic and asymptomatic patients. Neurosurgery 1988; 6:710-713.

15. Songer M, Ghosh L, Spencer D. Effects of sodium hyaluronate on peridural fibrosis after lumbar laminectomy and discectomy. Spine 1990; 15:550-554.

16. Key JA, Ford LT. Experimental intervertebral disc lesions. Journal Bone Joint Surgery (AM) 1948; 30:621-630.

17. Omnipaque ${ }^{\mathrm{TM}}$ product insert. Princeton, NJ, Nycomed, Inc., 1996.

18. Lasser EC, Lyon SG, Berry CB. Reports on contrast media reactions: Analysis of data from reports to the U.S. Food and Drug Administration. Radiology 1997; 203:605-610.

19. Ndosi BN, Ndosi NK, Kazema RR. Myelography with Omnipaque $^{\mathrm{TM}}$ (Iohexol) using basic radiographic facilities: The main adverse effects. Cent Afr J Med 1996; 42:192-195.

20. Kerkkemp HEM, Gielen MJM. Cardiovascular effects of epidural local anesthetics: Comparison of $0.75 \%$ bupivacaine and $0.75 \%$ ropivacaine, both with adrenaline. Anaesthesia 1991; 46:361-365.

21. Aristocort ${ }^{\mathrm{TM}}$ Forte Product Insert. Deerfield, IL, Fujisawa USA, Inc., 1994.

22. Abram SE, O’Connor TC. Complications associated with epidural steroid injections. Reg Anesth 1996; 21:149-162.

23. Jacobs S, Pullan PT, Potter JM et al. Adrenal suppression following extradural steroids. Anaesthesia 1983; 38:953-956.

24. Nelson DA, Vates TS, Thomas RB. Complications from intrathecal steroid therapy in patients with multiple sclerosis. Acta Neurol Scand 1973; 49:176-188.

25. Dougherty JH, Fraser RAR. Complications following intraspinal injections of steroids. J Neurosurg 1978; 48:1023-1025.
26. Shealy CN. Dangers of spinal injection without proper diagnosis. JAMA 1966; 197:156-158.

27. Watson D. Hyaluronidase. Br J Anaesth 1993; 71:422425.

28. Szabo G, Magyar S. Effect of hyaluronidase on capillary permeability, lymph flow, and passage of dyelabelled protein from plasma to lymph. Nature 1958; 182:377-379.

29. Hechter O, Dopkeen SK, Yudell MH. Clinical use of hyaluronidase in hypodermoclysis. J Pediat 1947; 30:645-656.

30. Hodgkin AL, Katz B. The effect of sodium ions on the electrical activity of the giant axon of the squid. $J$ Physiol (London) 1949; 108:37-77.

31. Hubbard JL, Jones SF, Landau EM. An examination of the effects of osmotic pressure changes upon transmitter release from mammalian motor nerve terminals. J Physiol (London) 1968; 197:639-657.

32. King JS, Jewett DL, Sundberg HR. Differential blockade of cat dorsal root $\mathrm{C}$ fibers by various chloride solutions. J Neurosurg 1972; 36:569-583.

33. Aldrete JA, Zapata JC, Ghaly R. Arachnoiditis following epidural adhesiolysis with hypertonic saline. Report of two cases. Pain Digest 1996; 6:638-370.

34. Racz GB, Holubec JT. Lysis of adhesions in the epidural space. In Racz GB (ed). Techniques of Neurolysis. Boston, Kluwer Academic Publishers 1989, pp 73-86.

35. Arthur J, Racz GB, Heinrich R et al. Epidural space: Identification of filling defects and lysis of adhesions in the treatment of chronic painful conditions. Abstracts of the $7^{\text {th }}$ World Congress on Pain, Paris, IASP Punlications, 1993, 557.

36. Racz GB, Heavner JE, Raj PP. Percutaneous epidural neuroplasty: Prospective one-year follow-up. Pain Digest 1999; 9:97-102.

37. Manchikanti L, Pakanati RR, Bakhit CE et al. Role of adhesiolysis and hypertonic saline neurolysis in management of low back pain: Evaluation of modification of the Racz protocol. Pain Digest 1999; 9:9196.

38. Heavner JE, Racz GB, Raj PP. Percutaneous epidural neuroplasty: Prospective evaluation of $0.9 \% \mathrm{NaCl}$ vs $10 \% \mathrm{NaCl}$ with or without hyaluronidase. Reg Anesth Pain Med 1999; 24:202-207. 